\title{
EFFECTS OF A NEW PEDESTRIAN PATHWAY IN \\ GRAND TETON NATIONAL PARK ON BREEDING SAGEBRUSH SONGBIRDS
}

\author{
ANNA CHALFOUN $\downarrow$ WyOMING COOPERATIVE FiSH \& WildLIFE RESEARCH UNIT \\ UNIVERSITY OF WYOMING $\downarrow$ LARAMIE
}

\section{$\downarrow \quad$ INTRODUCTION}

Human-induced changes to natural landscapes have become ubiquitous, resulting in exposure of wildlife populations to novel stressors (Munns 2006). While it is clear that changes such as habitat loss can directly impact wildlife species, less clear is the extent to which human presence itself functions as a disturbance that influences wildlife behaviors with important fitness consequences. Animals clearly respond to perceived risk of predation by natural predators via, for example, fleeing, or altering foraging and/or breeding habitat selection (Marzluff 1988, Hakkarainen et al. 2001, Frid and Dill 2002, Blumstein 2006, Borkowski et al. 2006, Fontaine and Martin 2006). Such responses can alter access to important resources, energy budgets, and therefore attributes such as body condition (Bechet et al. 2004) with potential impacts to survival and reproductive output. Of critical importance to the management of wildlife populations is therefore to determine 1) whether wildlife species perceive human presence as predation risk, 2) how individuals respond to such risk, and 3) how such responses influence fitness consequences and therefore population dynamics and community structure.

Birds are known to respond behaviorally to perceived predation risk (e.g., Marzluff 1988, Hakkarainen et al. 2001, Fontaine and Martin 2006, Chalfoun and Martin, in press), and may therefore respond similarly to human disturbance. Landbirds are an integral component of ecosystems and serve a wide range of ecological roles from pollinating plants to controlling insect populations. Construction of a bicycle pathway through portions of Grand Teton National Park in June, 2008 may influence landbird abundance, diversity, productivity, and/or breeding behavior, as numerous migratory landbird species breed within the lower elevation shrubsteppe and forest habitat through which the pathway will traverse.

This project was established in order to evaluate the potential impacts of pathway development and use on breeding landbird species. The overall goal of the work is to evaluate the impact of pathway development and use on breeding bird spatial and temporal habitat use, relative abundance, species composition and diversity, reproductive success, and breeding behavior. Specific objectives are to:

1. Identify avian species using project area habitats before, during, and after pathway construction and use.

2. Quantify the spatial and temporal habitat use of avian species within the project area before, during, and after pathway construction and use.

3. Based on objectives 1 and 2 , evaluate the effects of the pathway on avian abundance and community composition and diversity.

4. Document the breeding productivity (clutch size, hatching success, abandonment rates, nest success) of all observed non-game bird species in relation to the pathway.

5. Document site fidelity and breeding behavior patterns for a focal species, the Brewer's Sparrow (Spizella breweri; a declining, sagebrush-steppe obligate species) before, during and after pathway construction and use. 


\section{METHODS}

I am utilizing a Before-After/Control-Impact study design to quantify effects. Field efforts (before/control year) began during May - August, 2007, were replicated in May - August, 2008 during pathway construction, and will be repeated during two seasons of pedestrian use (2009 and 2010). Within sagebrush habitats along the highway and proposed pathway route between Moose and South Jenny Lake Junction, I randomly established three paired sets of 25ha study plots. One plot in each pair straddles the road and pathway route, and the other was placed in similar habitat/topography but $\geq 500 \mathrm{~m}$ from the transportation corridor. Data collected within control plots will serve to separate possible annual and habitat effects from experimental effects.

During each year and within each study plot, my crew and I are documenting avian abundance, diversity, and spatial habitat use using line transect surveys with distance estimation. Line transects are 1 $\mathrm{km}$ in length and centered within each control study plot in a randomly chosen direction. Experimental transects are oriented perpendicular to the road and pathway in order to quantify possible changes in avian metrics with increasing distance from the disturbed area. An additional study plot was established just north of the Moose entrance station to increase sample sizes for distance from road/path analyses.

To quantify reproductive success, we are intensively nest searching within each study plot. Nests are monitored every two days until completion (failure or fledging). Nest locations are recorded using GPS, and the distance to the road, path and nearest forest edge will serve as additional covariates in analyses. In order to determine whether pathway and/or road disturbance changes parental behaviors and/or investment with potential fitness ramifications, we are measuring egg/clutch mass, incubation behavior ( $\%$ attentiveness, length of on/off bouts), nestling size, and nestling feeding rates for the main focal species (Brewer's sparrow, Spizella breweri). Egg mass is recorded for nests with known initiation dates during days 1-3 of the incubation period using a portable electronic balance. We are documenting incubation behavior and nestling feeding rates via video taping of nests. Nestling mass, and tarsus and wing chord length is measured on day 5 of the nestling period in order to assess offspring quality. In order to more closely document potential territorial/nest site selection shifts of Brewer's sparrow's within and across seasons, we are target-netting and uniquely color-banding a sub-set
(5-10) of focal pairs to be intensively monitored during all four study years. Basic habitat structural measurements (shrub cover, height, and density and understory cover) was collected during July, 2007 to account for additional habitat effects and ensure similarity between paired study plots.

Abundance, diversity and productivity metrics will be compared between experimental and control plots within each of the four study years. As a result, I will generate comparisons for a pre-disturbance/control year, and two types of human disturbance (pathway construction and human use of the pathway). Year four data will help determine the extent to which the birds may acclimate to the disturbance generated by the pathway.

If the pathway construction and/or use constitutes a disturbance to which birds will respond, I predict that avian abundance, diversity, productivity, site fidelity and breeding behavior measurements should be similar among experimental and paired control plots during year 1 , and lower in experimental plots compared to controls during construction and/or use years. If the road constitutes a disturbance, metrics should differ (in a consistent direction) between the experimental versus control plots during all four years. If neither the road nor pathway has any effect, metrics should be similar between treatments during all four years. If both the road and pathway generate effects, the addition of the pathway should produce additional variance between the experimental versus control plots during years 2,3 , and/or 4 . Abundance and diversity data from the 4 transects straddling the path/road within sagebrush habitats will also be analyzed per $50-\mathrm{m}$ transect segment in an attempt to determine the distance to which possible disturbance effects permeate away from the pathway/road.

\section{$\uparrow \quad$ RESUlTS}

Data analysis from the 2008 field season is ongoing, and the following preliminary patterns should not yet be cited. Similar to 2007, avian abundance (all species combined), as indexed by the average number of detections per survey visit, did not differ between experimental and control plots (Paired T test, $t=-.60$, $\mathrm{df}=2, P=0.61)$, nor did the abundance of the most common species (Brewer's sparrow; $t=-.31, \mathrm{df}=2, P$ $=0.78$ ). Species richness (total number of species observed within a site) did not differ among treatments $(t=1.51, \mathrm{df}=2, P=0.27)$. Analyses within experimental sites focused on quantifying avian abundance/richness and other metrics in relation to distance from disturbance are not yet completed. 


\begin{tabular}{llll}
\hline Metric(s) & Method & Sample Sizes \\
\hline & & \\
Abundance, diversity & Transect surveys & 3 surveys completed within 7 plots \\
Nest success, clutch size & Nest searching/monitoring & 210 nests of 6 species* \\
Habitat shifts, site fidelity & Target netting/color banding of focal & 50 new BRSP captured/banded with no mortality \\
& pairs & \\
Egg mass & Weighing of eggs & 54 BRSP clutches measured \\
Incubation behavior & Video taping & 34 BRSP nests (136 video hrs.) \\
Nestling size/quality & Nestling measurements & 51 BRSP broods measured \\
Nestling feeding rates & Video taping & 42 BRSP nests (168 video hrs.) \\
& & \\
\hline
\end{tabular}

\footnotetext{
* Species studied:

Brewer's Sparrow (BRSP)

Vesper Sparrow

Green-Tailed Towhee

Chipping Sparrow

White-Crowned Sparrow

Horned Lark
}

Table 1. Summary of 2008 Field Activities.

Brewer's sparrow clutch size did not vary among experimental and control plots $(t=1.46, \mathrm{df}=2$, $P=0.28$ ). Mean BRSP egg mass was higher in the control sites for the two northern sets, but overall did not vary between treatments $(t=-1.53, P=0.27)$. Unlike 2007, however, BRSP hatching success in 2008 was lower within the road/pathway sites than the control sites ( $t=3.97, P=.05$; Figure 1). A similar (but insignificant) trend was observed for all species combined $(t=2.14, P=0.16)$. The lower hatching success associated with the road/pathway sites may have been related to changes in incubation behavior resulting from construction disturbance. I will be able to test this relationship when the behavioral (video) analyses are completed. Nest abandonment in experimental sites $(6.2 \%)$ was only slightly higher during pathway construction than during 2007 (5.3\%). Nestling mass did not differ among treatments overall, though there was a trend towards larger nestlings in the experimental plots in 2008 (Figure 2; Year $\mathrm{x}$ treatment interaction, $F=2.82, P=0.09$ ).

Nest predation rates, as assayed by daily nest survival probabilities, as in 2007 were again consistently lower within road/pathway sites compared to controls for all species combined (Figure 3). The trend was especially evident for Brewer's sparrows (Figure 4; Paired T test, $t=5.20, d f=2, P=0.04$ ).

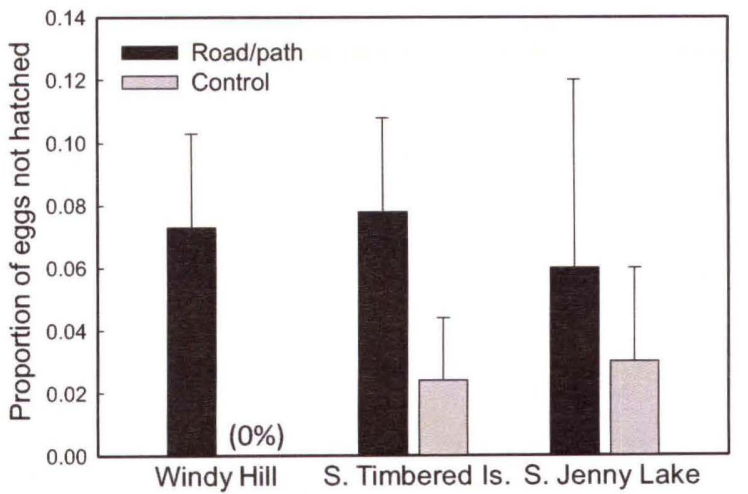

Figure 1. Hatching success of Brewer's sparrows in $\mathrm{road} /$ pathway versus paired control sites.

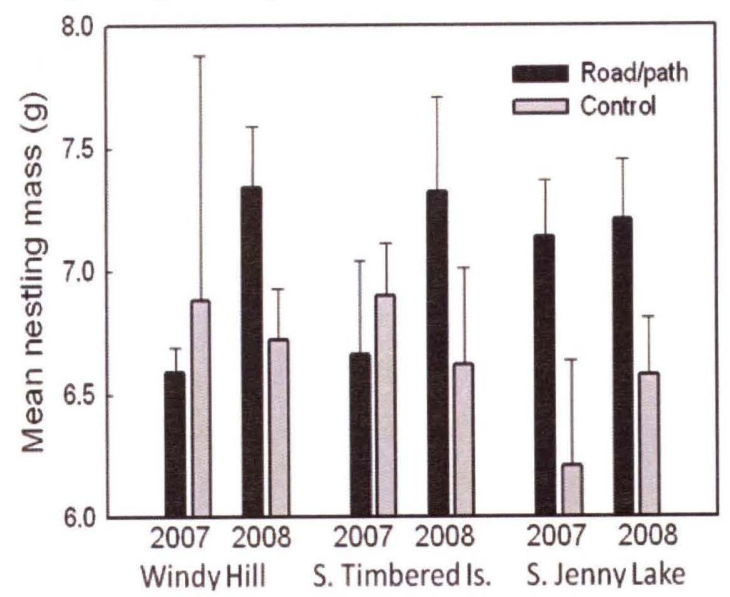

Figure 2. Mean nestling mass (g) at day 5 for Brewer's sparrows with a brood size of $n=3$ during 2007 and 2008 . 


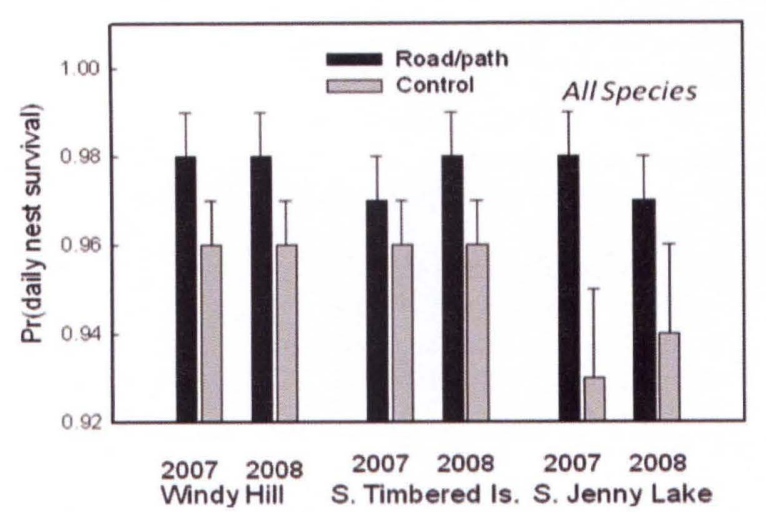

Figure 3. Avian (all species combined) nest success (probability of daily nest survival) within experimental versus control sites during 2007 and 2008.

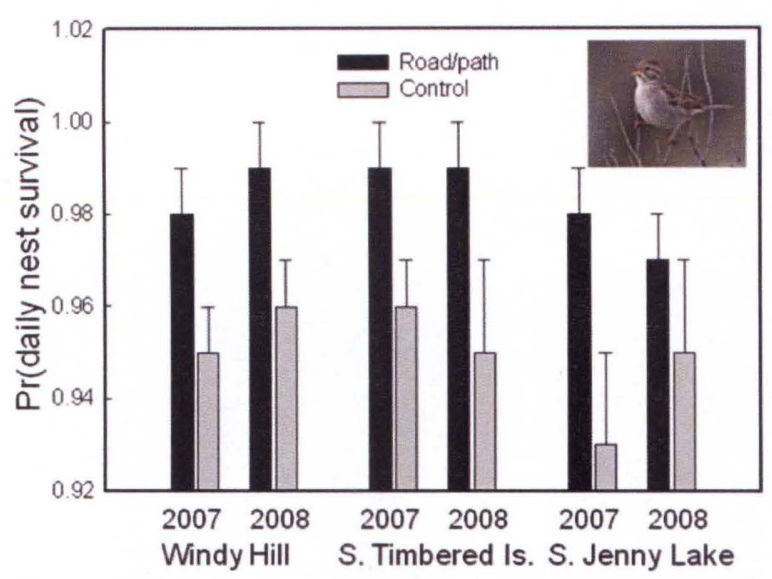

Figure 4. Brewer's sparrow nest success (probability of daily nest survival) within experimental versus control plots during 2007 and 2008.

Spring came much later in 2008 than in 2007, which delayed migratory bird arrival times and initiation dates of first nests, especially for Brewer's sparrows. For example, in 2008 the mean number of detections for BRSP/site during the first survey week in May was 2.2 compared to 12.5 in 2007 . Overall abundance (all species combined) was similar in 2008 (36.67 detections/survey visit/site) to 2007 (38.78), but the abundance of BRSPs in 2008 (7.06) remained at approximately half of that in 2007 (16.0). Mean clutch sizes were also slightly lower in 2008 for the three main study species (Figure 5). Birds may have altered settlement and nesting patterns in 2008 due to lingering snow pack and cold temperatures into June, 2008, which influenced food (insect) availability. I will carefully account for climatic variables in final analyses. Several 2008 nests containing 5-6 day-old nestlings failed due to heavy rains collapsing the nest. Two adult birds from two different nests were also found dead following a hail event.

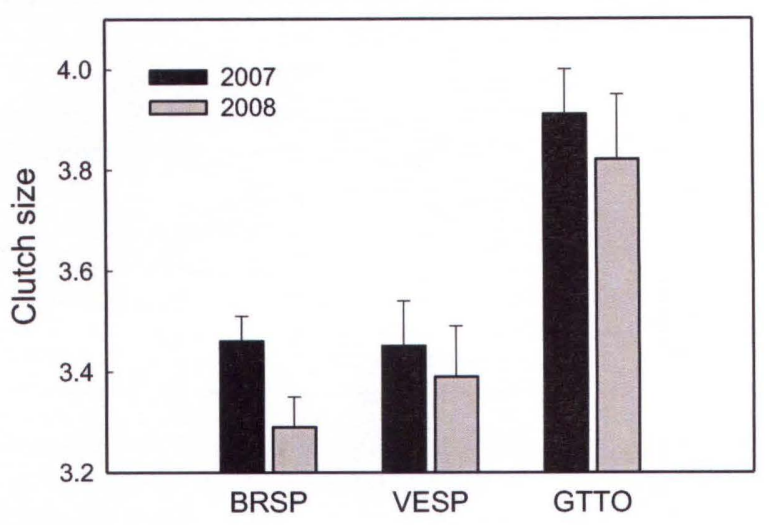

Figure 5. Mean clutch sizes $( \pm 1 \mathrm{SE})$ of the three most abundant songbird species (Brewer's sparrow, vesper sparrow, green-tailed towhee) during 2007 and 2008.

\section{$\uparrow \quad$ DISCUSSION}

Because data collection and analysis for the project are on-going, conclusions about potential impacts of the new pathway on the songbird community would be premature. Data collection will be repeated in 2009 and 2010 using identical methods and sampling protocols to those in 2008 and 2007, in order to compare avian responses during pedestrian use of the path to the pre-construction and construction years. Resightings of color-banded Brewer's sparrows will continue to serve as a barometer of site fidelity among the two study treatments.

Preliminary analyses do not suggest a major influence of pathway construction on songbird abundance, diversity, nest success, or investment in offspring; though incubation rhythms may have been affected as assayed by the lower hatching rates and slight increase in nest abandonment. Offspring size (nestlings at day 5) seemed to parallel nest predation risk rather than human disturbance. Nestling feeding rates were therefore likely not significantly influenced by construction activities, though the completion of the video and within-plot distance from the road/path analyses are needed for verification. Construction effects may have been minimized by the fact that experimental sites were not consistently exposed to construction crews and equipment throughout the whole nesting season.

The repeated observation that nest predation rates were consistently higher away from the road corridor is intriguing. Anecdotally, we observed more diurnal rodents (e.g., chipmunks) that may be important nest predators within control plots. One potential 
hypothesis for the nest predation patterns is therefore that the predators (coyotes, raptors, etc.) of these small mammal species may be preferentially utilizing (or more abundant along) the transportation corridor. I will be testing this hypothesis via predator surveys and timelapse infra-red video monitoring of a sub-set of Brewer's sparrow nests this summer.

\section{$\uparrow$ LiTERATURE Cited}

Bechet, A., J.F. Giroux and G. Gauthier. 2004. The effects of disturbance on behaviour, habitat use and energy of spring staging snow geese. Journal of Applied Ecology 41:689-700.

Blumstein, D.T. 2006. Developing an evolutionary ecology of fear: how life history and natural history traits affect disturbance tolerance in birds. Animal Behaviour 71:389-399.

Borkowski, J.J., P.J. White, R.A. Garrott, T. Davis, A.R. Hardy and D.J. Reinhart. 2006. Behavioral responses of bison and elk in Yellowstone to snowmobiles and snow coaches. Ecological Applications 16:19111925.
Chalfoun, A.D. and T.E. Martin. Habitat structure mediates predation risk for sedentary prey: experimental tests of alternative hypotheses. In press. Journal of Animal Ecology. Fontaine, J.J. and T.E. Martin. 2006. Parent birds assess nest predation risk and adjust their reproductive strategies. Ecology Letters 9:428-434.

Frid, A. and L. Dill. 2002. Human-caused disturbance stimuli as a form of predation risk. Conservation Ecology 6:1-11.

Hakkarainen, J., P. Ilmonen, V. Koivunen and E. Korpimaki. 2001. Experimental increase of predation risk induces breeding dispersal of Tengmalm's owl. Oecologia 126:355-359.

Marzluff, J.M. 1988. Do pinyon jays alter nest placement based on prior experience? Animal Behaviour 36:1-10.

Munns, W.R. Jr. 2006. Assessing risks to wildlife populations from multiple stressors: overview of the problem and research needs. Ecology and Society 11:1-23. 